\title{
DESIGN OPTIMIZATION FOR A PASSIVE MESH SCREEN WAVE ABSORBER FOR THE CCOB
}

\author{
Christian Klinghammer ${ }^{1}$, Pedro Lomónaco Tonda ${ }^{1}$ and Pablo Higuera Caubilla ${ }^{1}$
}

\begin{abstract}
A new passive wave absorber, consisting of multiple mesh screens, for the Cantabria Coastal and Ocean Basin (CCOB) was optimized regarding its overall length and performance. Physical model wave flume tests were conducted to calibrate and verify a 2D-RANS model utilizing different combinations of vertical and horizontal mesh screens. The final design of the main wave absorber at the back wall of the new CCOB was obtained by simulating different mesh screen configurations at full scale using the IH-2VOF model. Very good wave absorption over the entire working range of the wave basin could be achieved with a relatively short passive mesh screen wave absorber.
\end{abstract}

Keywords: passive wave absorber; wave basin; $I H-2$ VOF

\section{INTRODUCTION}

\section{CCOB}

The Cantabria Coastal and Ocean Basin is a multidirectional wave basin for shallow and deep water physical model testing. It is $44 \mathrm{~m}$ wide, $30 \mathrm{~m}$ long and $4.5 \mathrm{~m}$ deep, with operational water depths ranging from 0.2 to $3.0 \mathrm{~m}(11 \mathrm{~m}$ in the center pit). The wave basin features omnidirectional current generation and a segmented wavemaker with active wave absorption, consisting of 64 hydraulically driven wave boards on one side of the basin. The remaining three reflective basin walls had to be equipped with wave absorbers to minimize wave energy reflection from the boundaries or from model structures during model testing, see Fig. 1.

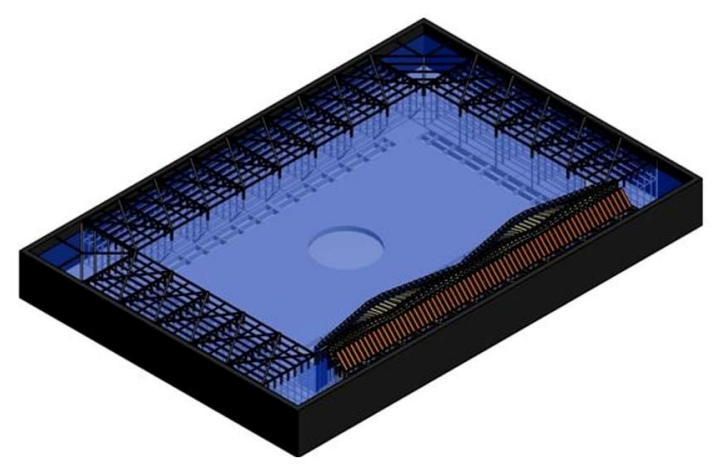

Figure 1. Schematic view of the Cantabria Coastal and Ocean Basin.

Passive wave absorbers consisting of several layers of vertical mesh screens (Jamieson and Mansard 1987) have been found to be very well suited for this purpose, due to their proven efficiency for different water depths and wave conditions, as to be simulated in the new wave basin, and the robustness of this kind of wave energy absorber.

The back wall wave absorber opposite of the wavemaker, receiving the majority of the generated wave energy, was optimized regarding its overall length and performance in order to maximize the wave absorption efficiency for the entire working range of the wave basin and to minimize the loss of valuable test area.

The design optimization involved physical model testing of prototype scale mesh screens in the wave-current flume at the University of Cantabria. This was followed by numerical model simulations of a 1:1 scale longitudinal cross-section of the CCOB equipped with different combinations of mesh screens, using IH-2VOF, a 2D RANS model with VOF free surface tracking.

\footnotetext{
${ }^{1}$ Environmental Hydraulics Institute IH Cantabria, University of Cantabria, Santander, 39011, Spain
} 


\section{Passive Mesh Screen Wave Absorber}

The wave absorber design, as proposed by Jamieson and Mansard, includes several layers of vertical mesh screens with decreasing porosity and spacing in between them from the front to the rear, with screen locations corresponding approximately to node locations of partial standing waves. They also stated that the optimum wave absorber length lies between $0.35 \mathrm{~L}$ and $1.0 \mathrm{~L}$, with $L$ being the maximum wave length to be absorbed. Taking into account the varying working conditions of the new wave basin and the resulting multitude of possible wave lengths to be attenuated, the new multi layer wave absorber would have varying wave absorption efficiencies for different wave conditions.

An initial design for the passive wave absorber for the new CCOB, following these recommendations, consisted of 8 layers of mesh screens with an overall length of $l=6 \mathrm{~m}$. While this design obeyed the optimum overall length ratio given by Jamieson and Mansard, it was incompatible with the wave basin's current outlets in the back, see Fig. 2.

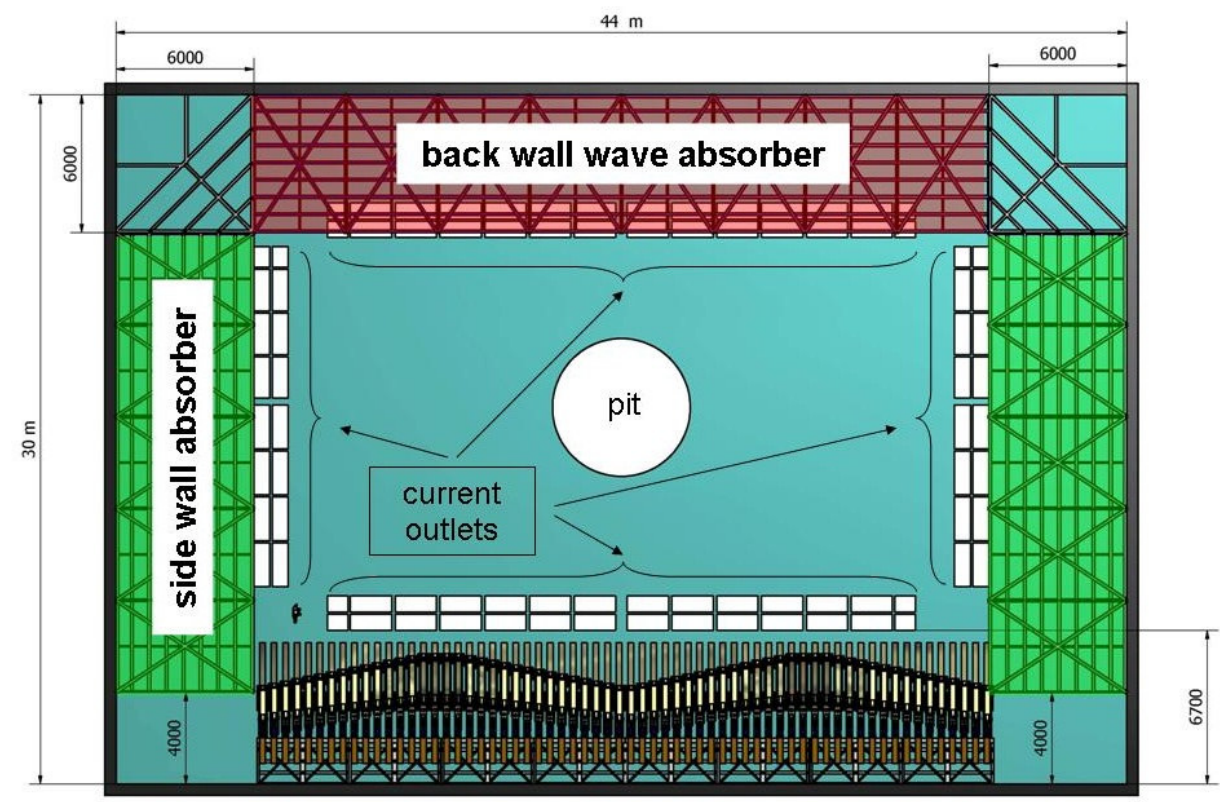

Figure 2. Reflective boundaries equipped with preliminary design wave absorber (8 layers, l=6 m).

In order to allow free current outflow the proposed design for the back wall wave absorber was adapted by the supplier, limiting the first two layers of mesh screens to water depths bigger than $h=2 \mathrm{~m}$, see Fig. 3 .

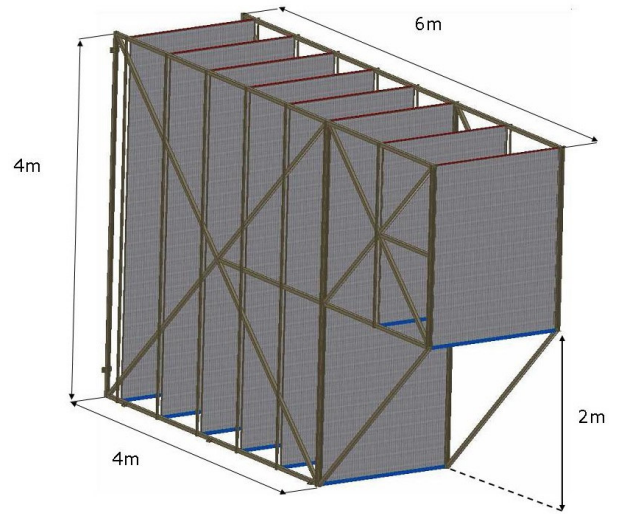

Figure 3. Adapted preliminary design for the back wall wave absorber.

This design had several disadvantages like poor performance in water depths lower than $2 \mathrm{~m}$ (especially with longer wave lengths), hard-to-predict behavior at water depth's around $2 \mathrm{~m}$ and difficult access of the current outlets with the wave absorbers installed in the wave basin. 
The design optimization process therefore focused only on the back wall wave absorber, trying to achieve high quality wave energy absorption with a maximum overall length of $l=4.5 \mathrm{~m}$, which is the distance of the back wall to the current outlets, and a minimum of 8 layers, covering the entire wave basin depth.

\section{DESIGN OPTIMIZATION}

The methodology during the physical and numerical testing of different combinations of mesh screen layers is shown in Fig. 4.

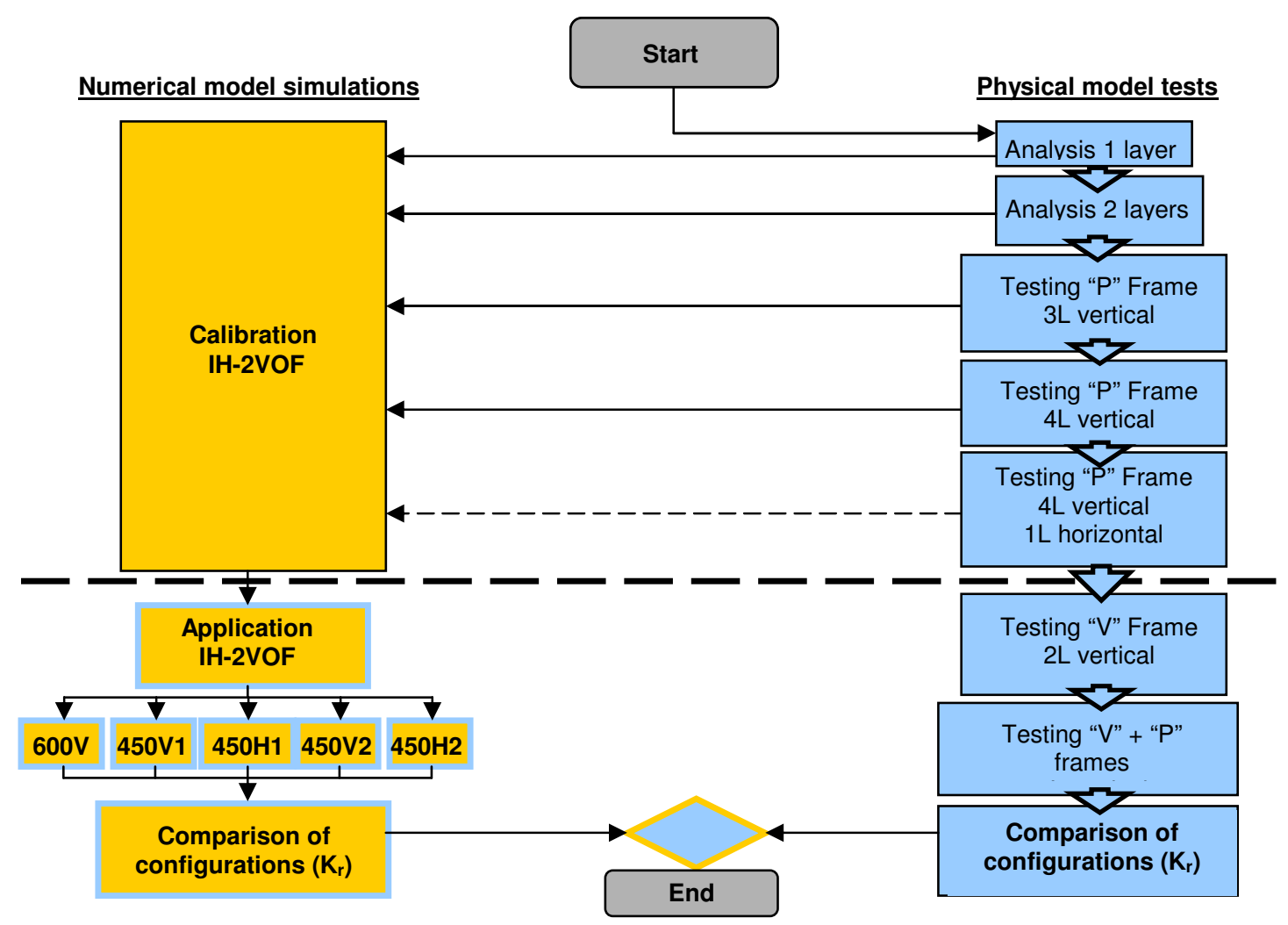

Figure 4. Methodology flow chart.

\section{Physical Model Tests}

In order to calibrate the IH-2VOF model, which was used for the 1:1 scale simulations of a longitudinal cross-section of the $\mathrm{CCOB}$, extensive physical model tests have been conducted at the wave-current flume of the University of Cantabria, which is $24 \mathrm{~m}$ long, $0.6 \mathrm{~m}$ wide and $0.75 \mathrm{~m}$ high and features a hydraulically driven piston-type wave maker with active wave absorption. The model tests were divided into three phases:

1. Characterization of prototype scale expanded metal screens (FILS) in the center of the wavecurrent flume.

2. Testing of several combinations of vertical and horizontal mesh screens arranged in parallel ("P") at the back of the wave-current flume.

3. Testing of several combinations of vertical mesh screens arranged in wedge shape ("V").

Steel frames were built to arrange the FILS expanded metal mesh screens in parallel and in wedge shape. These frames allowed for free positioning and were fixed to the carrier rails on top of the wavecurrent flume with clamps. The geometrical characteristics of the FILS screens are shown in Figure 5 and Table 1. All the wave tests were conducted with active wave absorption at the wavemaker and the free surface elevation was measured with up to $11 \mathrm{DHI}$ wave gauges. 


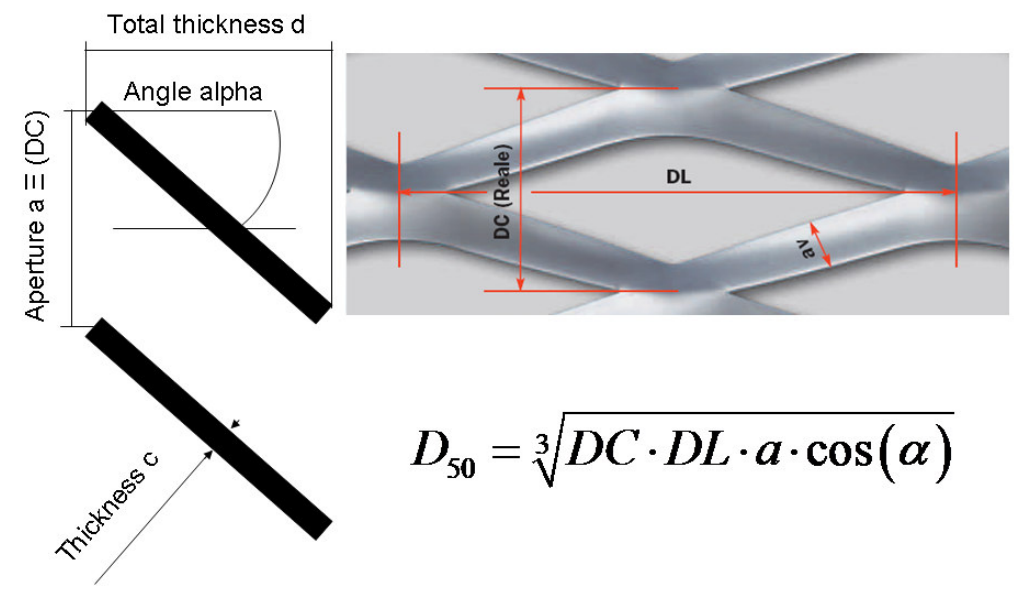

Figure 5. Geometrical definitions of FILS expanded metal screens.

\begin{tabular}{|c|c|c|c|c|c|c|c|}
\hline Name & Porosity & $\mathrm{DL} \times \mathrm{DC}-\mathrm{av} \times \mathrm{sp}[\mathrm{mm}]$ & $\mathrm{d}[\mathrm{mm}]$ & $\mathrm{a}[\mathrm{mm}]$ & $\mathrm{c}[\mathrm{mm}]$ & $\alpha[\stackrel{0}{\alpha}]$ & $\mathrm{D} 50[\mathrm{~mm}]$ \\
\hline Grafica & $51.5 \%$ & $100 \times 40(34)-10 \times 2$ & 15 & 34 & 2 & 41.41 & 47 \\
\hline Gate & $42.0 \%$ & $76 \times 31(35)-11 \times 2$ & 14 & 35 & 2 & 50.48 & 38 \\
\hline Fils5 & $36.2 \%$ & $62.5 \times 20(20)-7.5 \times 2$ & 10 & 20 & 2 & 48.19 & 26 \\
\hline Esperia & $23.3 \%$ & $100 \times 40(34)-15 \times 2$ & 13 & 34 & 2 & 64.32 & 39 \\
\hline Omega & $15.0 \%$ & $160 \times 40(52)-24 \times 2$ & 16 & 52 & 2 & 70.52 & 48 \\
\hline Reserve & $10.0 \%$ & $90 \times 30(38)-18 \times 2$ & 13 & 38 & 2 & 68.83 & 33 \\
\hline Privacy & $5.3 \%$ & $62.5 \times 20(28)-14 \times 2$ & 8 & 28 & 2 & 73.40 & 22 \\
\hline
\end{tabular}

In addition to the geometrical characteristics, the porous media characteristics of these seven types of expanded metal mesh screens (added mass coefficient, $\alpha, \beta$ ) to represent them correctly in the numerical model, were calibrated in numerical simulations of the first phase of the physical model testing, consisting of 11 different monochromatic and irregular wave sets with parallel arrangements of one and two identical screens in the center of the wave current flume and comparing the results with the obtained laboratory test data.

The obtained porous media characteristics were verified during numerical simulations of the second phase of the model testing, having up to four different vertical screens arranged in parallel at the back of the wave-current flume. During these tests the porosities of the screens decreased from the front to the rear of the screen configurations and the apertures of the expanded metal screens faced upwards towards the wavemaker. The effectiveness of an additional horizontal screen set at $0.8 \mathrm{~h}$ (Liu et al. 2007) was also tested during this test phase. The wave conditions for the testing of phase 2 and 3 are shown in Table 2, indicating monochromatic waves as $m$, irregular waves as $i$ and solitary waves as $s$, while the test set up for the parallel arrangements is shown in Figure 6. Table 3 shows the final results of the porous media characterization of the seven FILS expanded metal screens.

\begin{tabular}{|c|c|c|c|c|c|c|c|c|c|c|c|c|}
\hline \multicolumn{10}{|c|}{ Table 2. Tested wave conditions during physical model test phases 2 and 3. } \\
\hline Case & $\mathrm{h}$ & $\mathrm{H}, \mathrm{H}_{\mathrm{m}} 0$ & $\mathrm{~T}, \mathrm{Tp}$ & $\mathrm{u}_{\max }$ & $\mathrm{v}_{\max }$ & $\mathrm{C}$ & $\mathrm{Dur}$ & $\mathrm{dt}$ & $\mathrm{L}$ & $\mathrm{kh}$ & $\mathrm{h} / \mathrm{L}$ & $\mathrm{H} / \mathrm{h}$ \\
& {$[\mathrm{m}]$} & {$[\mathrm{m}]$} & {$[\mathrm{s}]$} & {$[\mathrm{m} / \mathrm{s}]$} & {$[\mathrm{m} / \mathrm{s}]$} & {$[\mathrm{m} / \mathrm{s}]$} & {$[\mathrm{s}]$} & {$[\mathrm{Hz}]$} & {$[\mathrm{m}]$} & & & \\
$\mathrm{m} 1$ & 0.4 & 0.05 & 1.4 & 0.14 & 0.11 & 1.71 & 90 & 60 & 2.39 & 2.63 & 0.167 & 0.125 \\
$\mathrm{~m} 2$ & 0.4 & 0.10 & 2.0 & 0.26 & 0.15 & 1.85 & 120 & 60 & 3.69 & 1.70 & 0.108 & 0.250 \\
$\mathrm{~m} 3$ & 0.4 & 0.15 & 3.0 & 0.38 & 0.15 & 1.92 & 170 & 60 & 5.77 & 1.09 & 0.069 & 0.375 \\
$\mathrm{~m} 4$ & 0.4 & 0.18 & 2.0 & 0.47 & 0.28 & 1.85 & 120 & 60 & 3.69 & 1.70 & 0.108 & 0.450 \\
$\mathrm{i} 1$ & 0.4 & 0.05 & 1.4 & - & - & - & 300 & 60 & 2.39 & 2.63 & 0.167 & 0.125 \\
$\mathrm{i} 2$ & 0.4 & 0.10 & 3.0 & - & - & - & 300 & 60 & 5.77 & 1.09 & 0.069 & 0.250 \\
$\mathrm{~s} 1$ & 0.4 & 0.10 & - & 0.44 & - & 2.22 & 60 & 60 & 18.35 & 0.14 & 0.022 & 0.250 \\
\hline
\end{tabular}



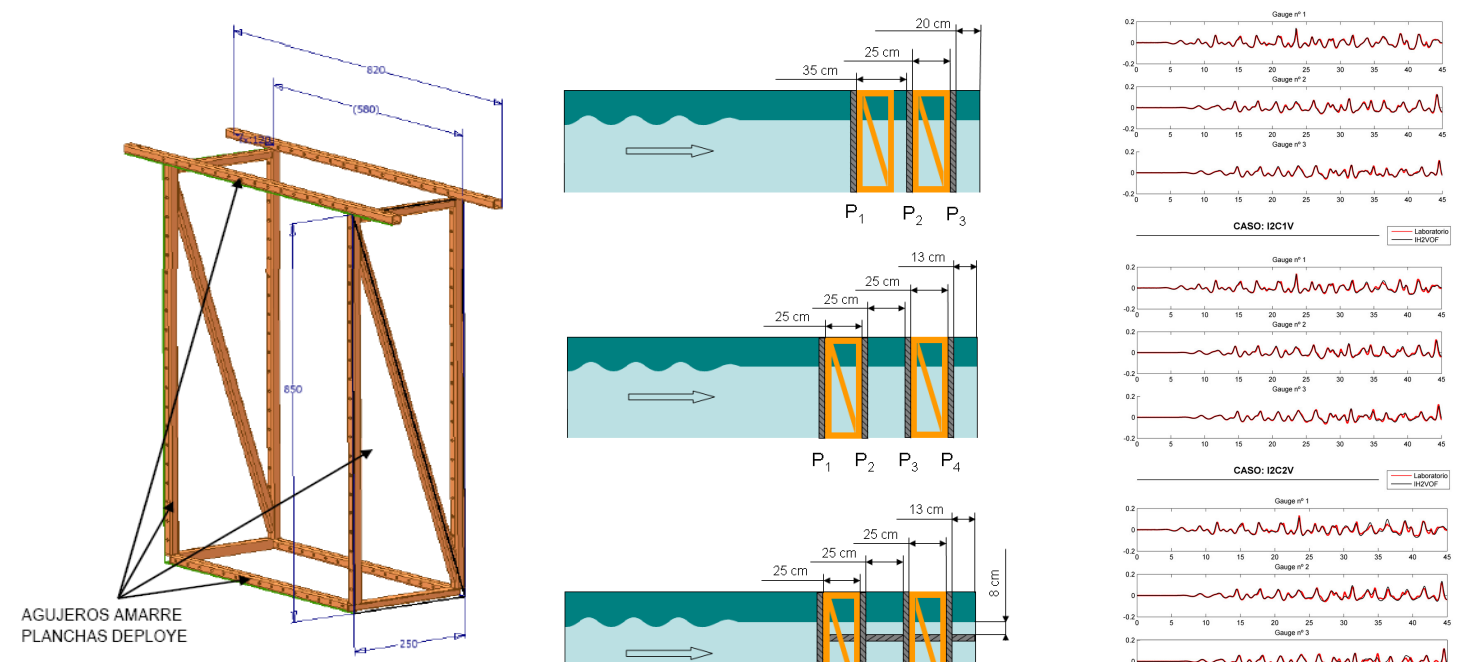

CASO: 12 CIV

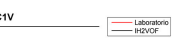
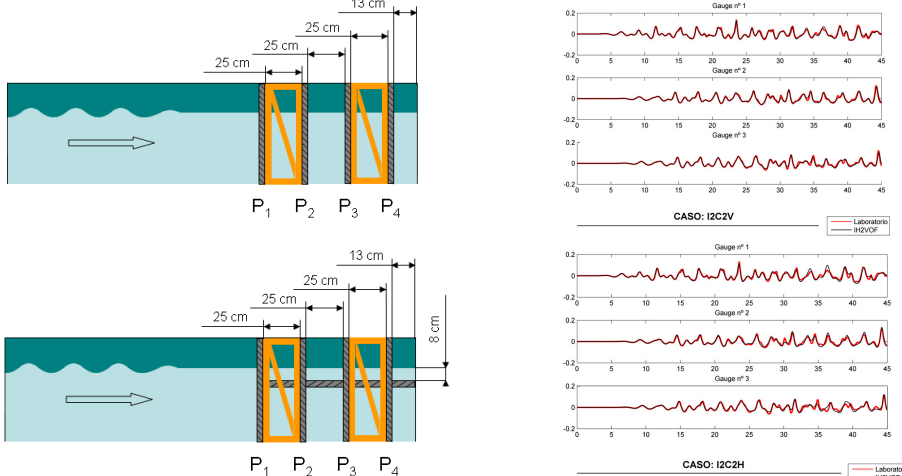
CASO: 12C2H

Figure 6. "P" frame and test set-up during physical model testing phase 2 (left and center), comparison of test data and numerical simulation data (right).

\begin{tabular}{|c|c|c|c|c|c|}
\hline Type & $\mathrm{P}[\%]$ & $\mathrm{D} 50[\mathrm{~mm}]$ & Added mass coef. & $\mathrm{FL}[\alpha]$ & FNL $[\beta]$ \\
\hline Grafica & 51.50 & 47 & 0.34 & 200.0 & 0.01 \\
\hline Gate & 42.00 & 38 & 0.34 & 200.0 & 0.3 \\
\hline Fils5 & 36.20 & 26 & 0.34 & 200.0 & 0.2 \\
\hline Esperia & 23.30 & 39 & 0.34 & 200,0 & 0.2 \\
\hline Omega & 15.00 & 48 & 0.34 & 200.0 & 0.2 \\
\hline Reserve & 10.00 & 33 & 0.34 & 200.0 & 0.1 \\
\hline Privacy & 5.30 & 22 & 0.34 & 200.0 & - \\
\hline
\end{tabular}

After finishing the characterization of the prototype mesh screens as porous media for the application of the IH-2VOF model at 1:1 CCOB scale, a third, additional phase of physical model testing was conducted to analyze the influence of the first (two) layers of mesh screen being arranged in a wedge shape. The theoretical improvement of wave absorption, having the first layers of mesh screen nonperpendicular to the wave front was already mentioned by Jamieson and Mansard.

Although such an arrangement of mesh screens could not be simulated using the IH-2VOF model, due to the highly three dimensional flow processes involved, several tests were conducted in the wavecurrent flume using a wedge frame for up to two mesh screens in front of the parallel frames. During testing, this configuration of mesh screens (only wedge and wedge plus parallel frame) indicated a superior performance than just the parallel arrangements. Due to the fact, that the incident wave angle could not be changed in the wave-current flume experiments, the effect of the wedge angle to incident wave angle could not be determined. Figure 7 shows the combinations of parallel and wedge frames tested, as well as some of the differences in working principles observed during the tests. It was also found that with the wedge shape in front, the ripples (generated by water flushing out of the wave absorber towards the wavemaker after the waves are reflected by the back wall of the flume) are smaller than compared to the parallel configurations. 


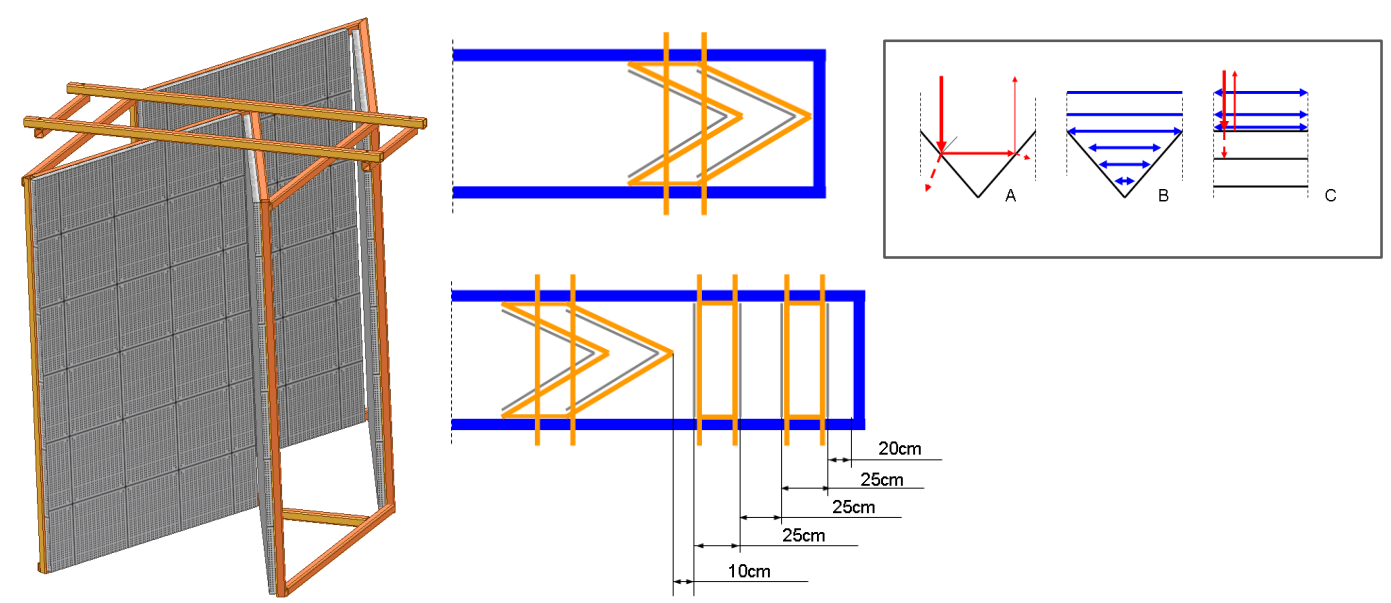

Figure 7. Wedge shape frame used during physical model testing phase 3, comparison "P" and "V" (right).

With the porous media characteristics of the FILS expanded metal screens obtained during phase 1 and 2 of the physical model testing, the calibrated IH-2VOF model, which showed a very good agreement in comparison to the laboratory test data, could be used to simulate a longitudinal cross-section of the CCOB with different combinations of mesh screens.

\section{1:1 CCOB Scale Simulations using IH-2VOF}

The suitability and accuracy of the IH-2VOF model for simulations of large scale laboratory experiments has been sown in the past (Lara et al. 2006). A complete longitudinal cross-section of the $\mathrm{CCOB}$ was modeled to simulate different configurations of vertical and horizontal mesh screens to be placed anywhere in the rear $6 \mathrm{~m}$ of the wave basin, see Fig. 8 .

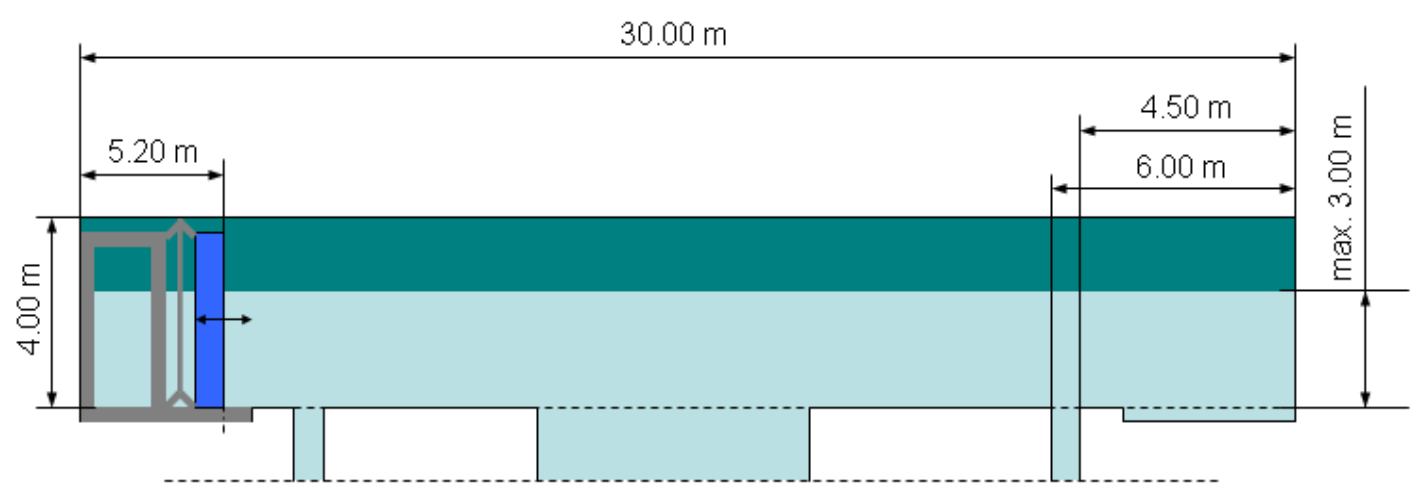

Figure 8. CCOB longitudinal cross-section.

After preliminary simulations of possible screen combinations, 5 different configurations of vertical and horizontal mesh screens were selected for comparison of its absorption efficiency in 18 different wave conditions, which represent different working conditions of the new wave basin. The main objective was to determine if a shortened passive wave absorber with an overall length of $l=4.5 \mathrm{~m}$, not disturbing the current outlets in the back of the basin, could be as efficient as the initially proposed design with an overall length of $l=6.0 \mathrm{~m}$. Therefore, the first of the 5 different screen configurations to be simulated was an exact copy of the $6 \mathrm{~m}, 8$ layer design $(600 \mathrm{~V} 1)$.

The first alteration to this concept was a shortened version with $l=4.5 \mathrm{~m}$, maintaining the same number of 8 vertical screens without additional horizontal screens (450V1). The third configuration consisted of the same vertical mesh screens as $450 \mathrm{~V} 1$ and $600 \mathrm{~V} 1$, but had additional horizontal mesh screens at $h=0.8 \mathrm{~m}, h=1.6 \mathrm{~m}$ and $h=2.4 \mathrm{~m}(450 \mathrm{H} 1)$. Configuration four consisted of a $4.5 \mathrm{~m}, 9$ vertical layer design without additional horizontal screens (450V2), while the last configuration had again three horizontal screens at the already mentioned positions $(450 \mathrm{H} 2)$. The complete descriptions of the porosities $n$ of each layer and its distance $d$ towards the back wall (v) or the basin floor respectively (h) of each configuration are given in Table 4, while the 18 wave conditions are shown in Table 5. 


\begin{tabular}{|c|c|c|c|c|c|c|c|c|c|c|}
\hline \multirow{2}{*}{ Config. } & \multicolumn{2}{|c|}{$650 \mathrm{~V} 1$} & \multicolumn{2}{|c|}{$450 \mathrm{~V} 1$} & \multicolumn{2}{|c|}{$450 \mathrm{H} 1$} & \multicolumn{2}{|c|}{$450 \mathrm{~V} 2$} & \multicolumn{2}{|c|}{$450 \mathrm{H} 2$} \\
\hline & $\mathrm{n}[\%]$ & $\mathrm{d}[\mathrm{m}]$ & $\mathrm{n}[\%]$ & $\mathrm{d}[\mathrm{m}]$ & $\mathrm{n}[\%]$ & $\mathrm{d}[\mathrm{m}]$ & $\mathrm{n}[\%]$ & $\mathrm{d}[\mathrm{m}]$ & $\mathrm{n}[\%]$ & $\mathrm{d}[\mathrm{m}]$ \\
\hline $1 \mathrm{v}$ & 51.5 & 6.00 & 51.5 & 4.50 & 51.5 & 4.50 & 51.5 & 4.50 & 51.5 & 4.50 \\
\hline $2 v$ & 51.5 & 5.00 & 51.5 & 3.50 & 51.5 & 3.50 & 42.0 & 3.70 & 42.0 & 3.70 \\
\hline $3 v$ & 42.0 & 4.00 & 42.0 & 2.70 & 42.0 & 2.70 & 36.2 & 3.00 & 36.2 & 3.00 \\
\hline $4 \mathrm{v}$ & 36.2 & 3.25 & 36.2 & 2.00 & 36.2 & 2.00 & 36.2 & 2.40 & 36.2 & 2.40 \\
\hline $5 v$ & 23.3 & 2.50 & 23.3 & 1.40 & 23.3 & 1.40 & 23.3 & 1.90 & 23.3 & 1.90 \\
\hline $6 \mathrm{v}$ & 23.3 & 1.75 & 23.3 & 0.90 & 23.3 & 0.90 & 23.3 & 1.40 & 23.3 & 1.40 \\
\hline $7 v$ & 15.0 & 1.00 & 15.0 & 0.50 & 15.0 & 0.50 & 15.0 & 0.95 & 15.0 & 0.95 \\
\hline $8 v$ & 10.0 & 0.25 & 10.0 & 0.20 & 10.0 & 0.20 & 10.0 & 0.55 & 10.0 & 0.55 \\
\hline $9 v$ & & & & & & & 10.0 & 0.25 & 10.0 & 0.25 \\
\hline $1 \mathrm{~h}$ & & & & & 23.3 & 2.40 & & 23.3 & 2.40 & 23.3 \\
\hline $2 \mathrm{~h}$ & & & & & 15.0 & 1.60 & & 15.0 & 1.60 & 15.0 \\
\hline $3 \mathrm{~h}$ & & & & & 10.0 & 0.80 & & 10.0 & 0.80 & 10.0 \\
\hline
\end{tabular}

\begin{tabular}{|c|c|c|c|c|c|c|c|c|c|c|}
\hline Case & $\mathrm{h}[\mathrm{m}]$ & $\begin{array}{c}\mathrm{H}, \mathrm{Hm} 0 \\
{[\mathrm{~m}]}\end{array}$ & $\begin{array}{c}\mathrm{T}, \mathrm{Tp} \\
{[\mathrm{s}]}\end{array}$ & $\begin{array}{l}\text { L, Lp } \\
\text { [s] }\end{array}$ & kh & $\mathrm{h} / \mathrm{L}$ & $\mathrm{H} / \mathrm{h}$ & $\begin{array}{l}u_{\max } \\
{[\mathrm{m} / \mathrm{s}]}\end{array}$ & $\begin{array}{l}{ }^{c} \max \\
{[\mathrm{m} / \mathrm{s}]}\end{array}$ & $\mathrm{C}[\mathrm{m} / \mathrm{s}]$ \\
\hline M1 & 3.0 & 1.000 & 3.00 & 12.69 & 1.49 & 0.236 & 0.333 & 1.15 & 1.04 & 4.23 \\
\hline M2 & 3.0 & 0.400 & 2.20 & 7.46 & 2.53 & 0.402 & 0.133 & 0.57 & 0.57 & 3.39 \\
\hline M3 & 3.0 & 0.400 & 3.00 & 12.69 & 1.49 & 0.236 & 0.133 & 0.46 & 0.41 & 4.23 \\
\hline 11 & 3.0 & 0.400 & 2.20 & 7.46 & 2.53 & 0.402 & 0.133 & - & - & - \\
\hline 12 & 3.0 & 0.400 & 3.00 & 12.69 & 1.49 & 0.236 & 0.133 & - & - & - \\
\hline S1 & 3.0 & 0.500 & 0.00 & 75.40 & 0.00 & 0.040 & 0.167 & 0.84 & - & 5.86 \\
\hline M4 & 2.0 & 0.660 & 2.45 & 8.46 & 1.49 & 0.236 & 0.330 & 0.93 & 0.84 & 3.45 \\
\hline M5 & 2.0 & 0.260 & 1.80 & 4.99 & 2.52 & 0.401 & 0.130 & 0.45 & 0.45 & 2.75 \\
\hline M6 & 2.0 & 0.260 & 2.45 & 8.46 & 1.49 & 0.236 & 0.130 & 0.36 & 0.33 & 3.45 \\
\hline 13 & 2.0 & 0.260 & 1.80 & 4.99 & 2.52 & 0.401 & 0.130 & - & - & - \\
\hline 14 & 2.0 & 0.260 & 2.45 & 8.46 & 1.49 & 0.236 & 0.130 & - & - & - \\
\hline S2 & 2.0 & 0.330 & 0.00 & 62.18 & 0.00 & 0.032 & 0.165 & 0.68 & - & 4.78 \\
\hline M7 & 1.0 & 0.330 & 1.74 & 4.25 & 1.48 & 0.235 & 0.330 & 0.66 & 0.59 & 2.45 \\
\hline M8 & 1.0 & 0.130 & 1.27 & 2.48 & 2.53 & 0.403 & 0.130 & 0.32 & 0.32 & 1.96 \\
\hline M9 & 1.0 & 0.130 & 1.74 & 4.25 & 1.48 & 0.235 & 0.130 & 0.26 & 0.26 & 2.45 \\
\hline 15 & 1.0 & 0.130 & 1.27 & 2.48 & 2.53 & 0.403 & 0.130 & - & - & - \\
\hline 16 & 1.0 & 0.130 & 1.74 & 4.25 & 1.48 & 0.235 & 0.130 & - & - & - \\
\hline S3 & 1.0 & 0.165 & 0.00 & 43.97 & 0.00 & 0.023 & 0.165 & 0.48 & - & 3.38 \\
\hline
\end{tabular}

The test conditions shown in Table 5 were selected to represent the working range of the CCOB, as mentioned earlier. Apart of testing different wave lengths, as the efficiency of this kind of passive wave absorber is strongly related to this wave characteristic, it was indented to generate increasing horizontal velocities. The resulting 18 wave states consisted of monochromatic waves (M1 to M9), irregular waves (I1 to I6) and solitary waves (S1, S2, S3), knowing that the absorption of very long waves, such as solitary waves, represents the hardest challenge for any kind of wave energy absorber. Groups of three monochromatic wave states, two irregular wave states and one solitary wave were simulated for three different water depths, trying to maintain comparability in between the different test conditions. For example M1 and M7 have the same non-dimensional wave characteristics $(k h, h / L, H / h)$ but differ in wave length and horizontal velocities, while M5 and M7 have similar wave lengths but differ in other characteristics, such as wave steepness. This kind of selection of test conditions allows for a better understanding of the effects of different design criteria, such as overall length or total number of vertical layers.

To compare the efficiency of the different mesh screen configurations the wave height based reflection coefficients $K_{r}$ were obtained using the free surface elevation at three positions in the center of the longitudinal cross-section for wave reflection analysis based on the Mansard and Funke method during the numerical simulations. In the case of solitary waves, the reflection coefficient $K_{r}$ was obtained with the simple relation of incident wave height to reflected wave height. 


\section{Comparison of Mesh Screen Combinations}

The 5 different configurations of expanded metal mesh screen were compared regarding their overall performance. A small reflection coefficient for just one or two test conditions does not necessarily mean that this configuration is best suited for installation in the new wave basin, bearing in mind the almost infinite possible combinations of wave characteristics over the entire working range. Figure 9 shows the comparison of the 5 mesh screen configurations tested with each of the 18 selected test conditions. The initial preliminary wave absorber design with an overall length of $l=6 \mathrm{~m}$ and 8 vertical layers of mesh screens is indicated in red as reference. As mentioned earlier, the main objective of this study was to determine if an optimized shortened passive wave absorber could reach the absorption efficiency of the initial longer design.

Comparing the 600V1 design with its shorter counterpart 450V1, the general negative effect of shortening the overall length becomes clearly visible. In almost all test conditions the $450 \mathrm{~V} 1$ design showed inferior wave absorption efficiency compared to the 600V1 design. The solitary wave cases, with their very long wave lengths, clearly indicate the influence of the wave absorber overall length, just as remarked by Jamieson and Mansard. The addition of the three horizontal screens of the $450 \mathrm{H} 1$ design helped to improve the efficiency of the shortened wave absorber in most of the test conditions, while in others they reduced the wave absorption.

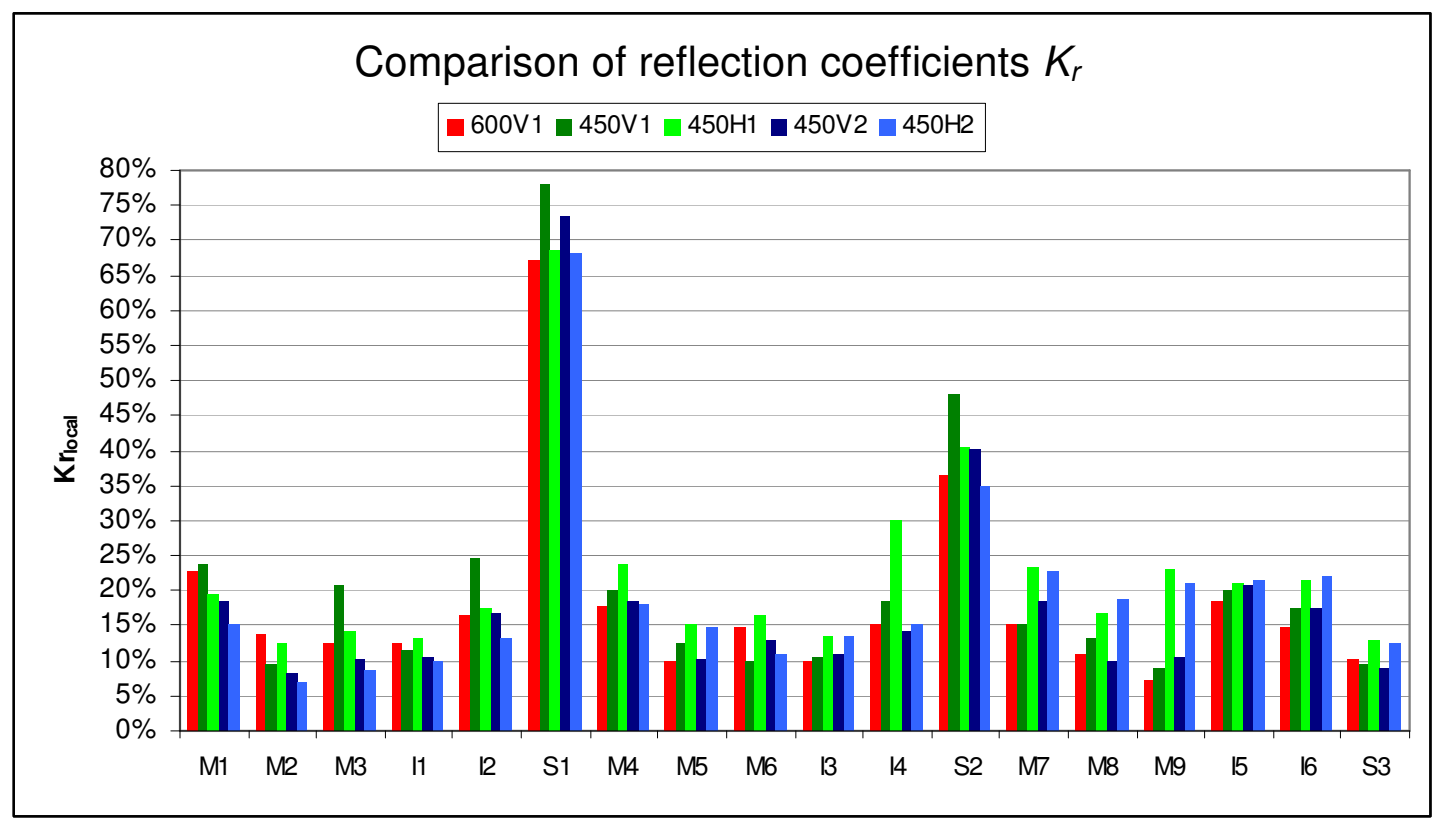

Figure 9. Comparison of reflection coefficients $K_{r}[\%]$ of the 5 simulated .mesh screen configurations.

The 4.5m, 9 vertical layer $450 \mathrm{~V} 2$ design showed big improvements compared to the $450 \mathrm{~V} 1$ design with 8 layers. Especially in the $3 \mathrm{~m}$ and $2 \mathrm{~m}$ water depths, this shortened design even outperforms the $600 \mathrm{~V} 1$ in most of the cases. The solitary wave cases S1 and S2 indicate very high wave reflection, but these test conditions only represent extreme cases for a better understanding of the relation between design and wave absorption efficiency. Again, the addition of three horizontal screens of design $450 \mathrm{H} 2$ reduced the wave reflection in some cases (especially the test conditions with longer wave lengths), but worsened the absorption efficiency in others.

\section{CONCLUSIONS}

A new passive wave absorber consisting of several layers of mesh screen, such as described by Jamieson and Mansard had to be design for the new CCOB. The preliminary design proposed by the supplier with an overall length of $1=6 \mathrm{~m}$ would have blocked the current outlets in the back of the wave basin. The adapted design with two different overall lengths fewer mesh screen in water depths below $h=2 \mathrm{~m}$ was considered disadvantageous. The simulation of different mesh screen configurations with the IH-2VOF model, which was calibrated with physical model tests using prototype scale mesh screens, allowed for a better understanding of the basic design principles of this kind of passive wave absorber. 
Although only normal wave incidence could be simulated with the $2 \mathrm{D}$ model, its application represents a huge improvement over the traditional design process for passive wave absorbers. It could be shown that a relatively short, optimized passive wave absorber can achieve the same or a better wave absorption efficiency than traditional longer designs. The $4.5 \mathrm{~m}, 9$ vertical layer design $450 \mathrm{~V} 2$ was found to be a better alternative to the proposed adapted design of the $600 \mathrm{~V} 1$ design, see Fig. 3 . The ratio of $l / L_{\max }(\mathrm{M} 1)$ of 0.354 still is within the recommendations given by Jamieson and Mansard.

The addition of horizontal screens could not be clearly be evaluated, since their influence seems ambiguous and has to be investigated further.

During the third phase of the physical model testing, the influence of the first (two) layers of mesh screen being arranged in a wedge shape was tested. The observed processes, together with the remarks by Jamieson and Mansard strongly encouraged an implementation of this detail into the final wave absorber design, although this could not be simulated and confirmed with the $2 \mathrm{D}$ numerical model. The influence of the wedge shape on the wave reflection of oblique waves could neither be simulated nor tested during this study.

The final design for the $4.5 \mathrm{~m}, 9$ vertical layer back wall wave absorber (with the $1^{\text {st }}$ layer in a wedge shape) together with the $6 \mathrm{~m}$ side wall wave absorbers (having the original $8^{\text {th }}$ layer removed) is shown in Figure 10. No horizontal screens are included in this design, but can be added easily in the future.

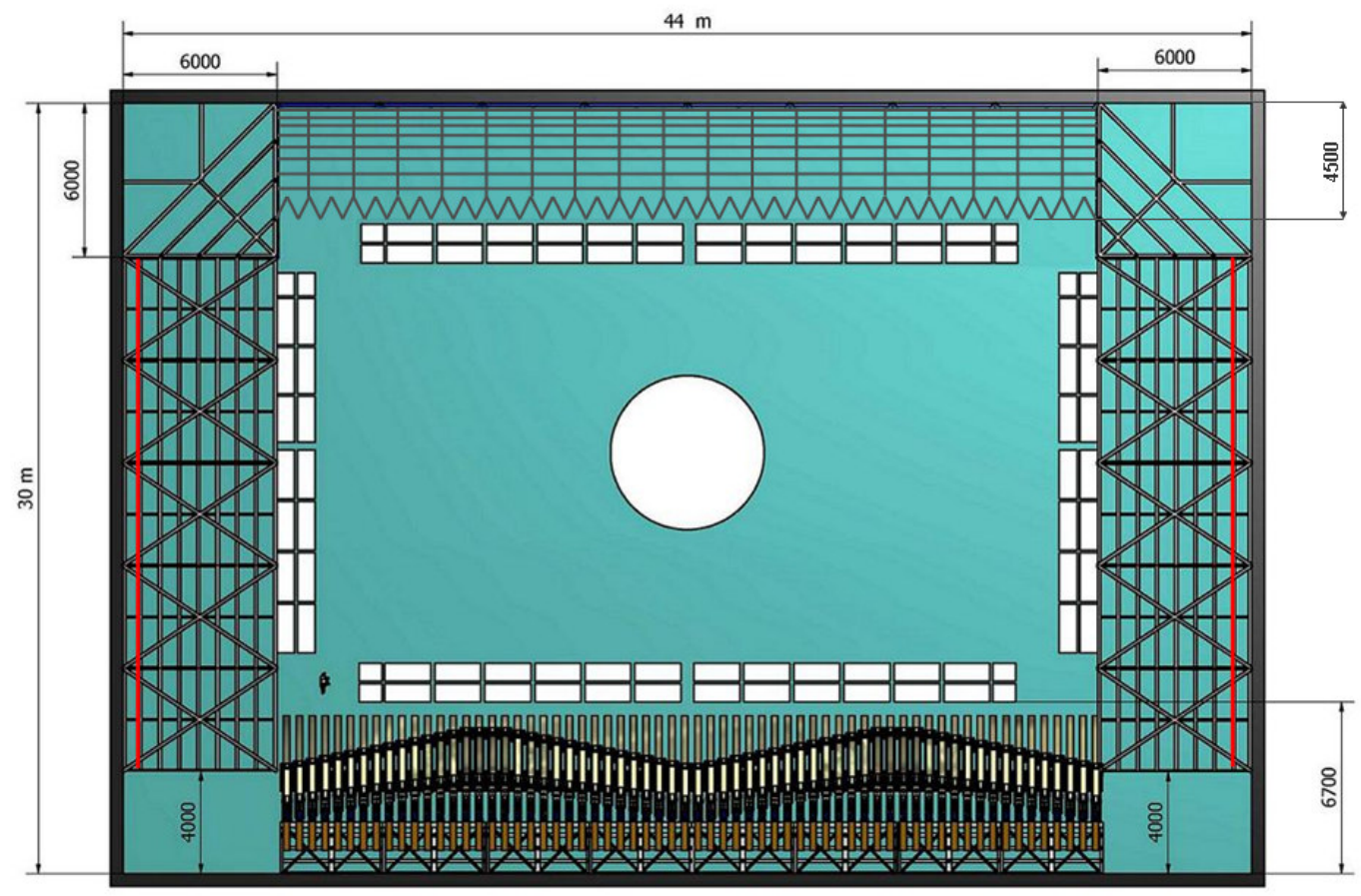

Figure 10. Final design for the passive wave absorber.

\section{Prototype Construction}

The conclusions of this study resulted in the construction of the prototype wave absorber for the CCOB. While the side wall wave absorbers were fixed to the basin, the back wall wave absorber was divided into 10 removable sections, see Fig. 11. Removing the back wall wave absorber allows for a bigger test area in cases were no wave absorption is necessary (construction of dissipative bathymetries for example). In first tests, the complete wave absorber showed an outstanding performance, which will be quantified in a detailed physical model test study, including oblique wave incidence and directional spreading. 

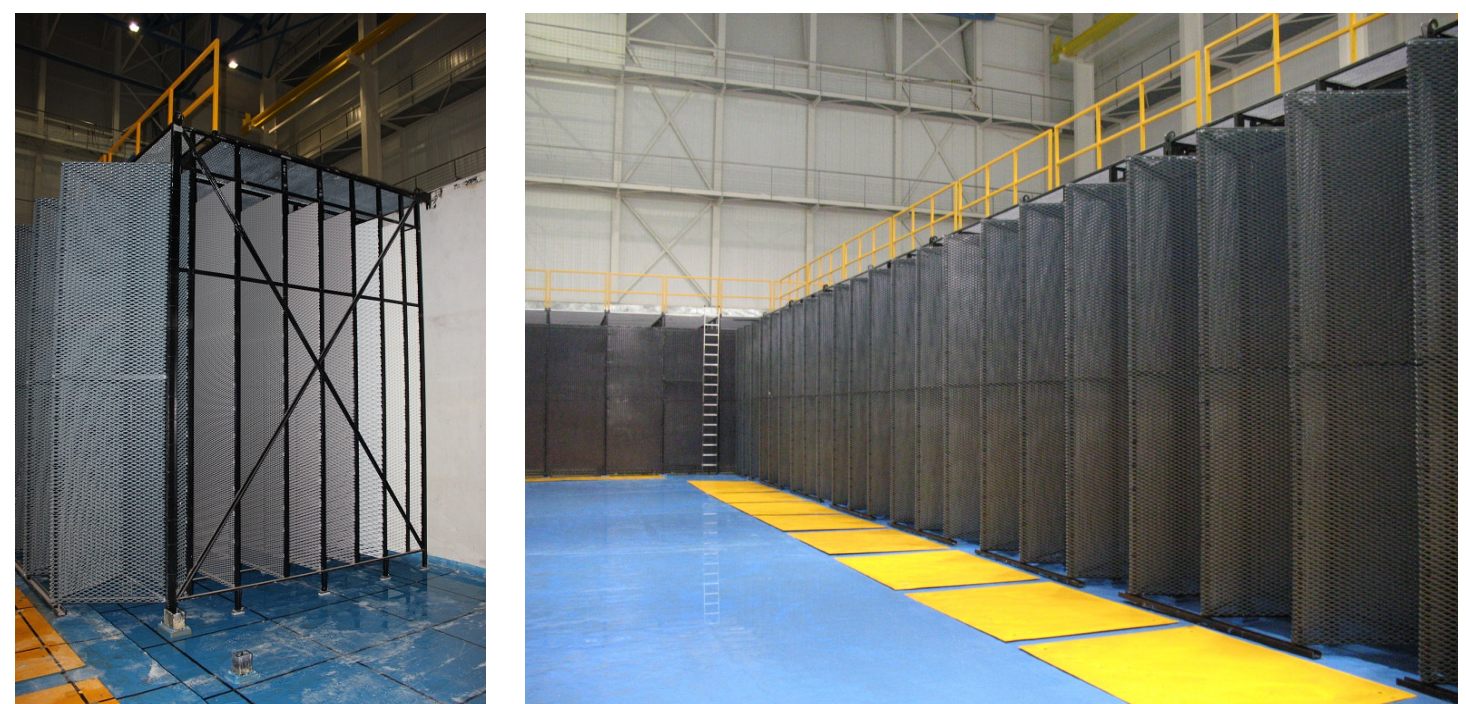

Figure 11. Back wall wave absorber at the CCOB, Left: single segment, Right: all segments installed

\section{REFERENCES}

Jamieson, W.W., and Mansard, E.P. 1987. An Efficient Upright Wave Absorber, Proceedings of Coastal Hydrodynamics '87, ASCE, 124-139.

Lara, J.L., Losada, I.J., Guanche, R., Vidal, C. 2007. Numerical and Experimental Investigation of Wave Interaction with Overtopped Vertical Breakwaters, Proceedings of the 30th International Conference on Coastal Engineering, ASCE, 4386-4387.

Liu, Y, Li, Y.C., Teng, B, 2007. Wave Interaction with a Perforated Wall Breakwater with a Submerged Horizontal Porous Plate, Ocean Engineering 34 (2007), 2364-2373. 